\title{
A novel phosphor for glareless white light-emitting diodes
}

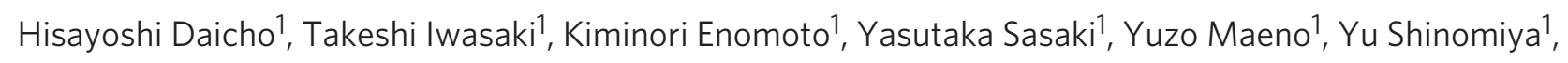
Shinobu Aoyagi ${ }^{2}$, Eiji Nishibori ${ }^{3}$, Makoto Sakata ${ }^{4}$, Hiroshi Sawa ${ }^{3}$, Satoru Matsuishi ${ }^{5} \&$ Hideo Hosono ${ }^{5}$

The luminous efficiency of white light-emitting diodes, which are used as light sources for next-generation illumination, is continuously improving. Presently available white lightemitting diodes emit with extremely high luminance because their emission areas are much smaller than those of conventional light sources. Consequently, white light-emitting diodes produce a glare that is uncomfortable to the human eye. Here we report a yellow-emitting phosphor, the $\mathrm{Eu}^{2+}$-doped chlorometasilicate $\left(\mathrm{Ca}_{1-x-y}, \mathrm{Sr}_{x}, \mathrm{Eu}_{y}\right)_{7}\left(\mathrm{SiO}_{3}\right)_{6} \mathrm{Cl}_{2}$, which can be used to create glareless white light-emitting diodes. The $\left(\mathrm{Ca}_{1-x-y}, \mathrm{Sr}_{x}, \mathrm{Eu}_{y}\right)_{7}\left(\mathrm{SiO}_{3}\right)_{6} \mathrm{Cl}_{2}$ exhibits a large Stokes shift, efficiently converting violet excitation light to yellow luminescence, and phosphors based on this host material have much less blue absorption than other phosphors. We used crystal structure analysis to determine the origin of the desired luminescence, and we used $\left(\mathrm{Ca}_{7-x-y}, \mathrm{Sr}_{x}, \mathrm{Eu}_{y}\right)_{7}\left(\mathrm{SiO}_{3}\right)_{6} \mathrm{Cl}_{2}$ and a blue-emitting phosphor in combination with a violet chip to fabricate glareless white light-emitting diodes that have large emission areas and are suitable for general illumination.

\footnotetext{
1 Department of Research and Development, Koito Manufacturing Co., Ltd, Shizuoka 424-8764, Japan. ${ }^{2}$ Department of Information and Biological Sciences, Nagoya City University, Nagoya 467-8501, Japan. ${ }^{3}$ Department of Applied Physics, Nagoya University, Nagoya 464-8603, Japan. ${ }^{4}$ SPring8/JASRI, Kouto, Sayo, Hyogo 679-5198, Japan. ${ }^{5}$ Frontier Collaborative Research Center, Tokyo Institute of Technology, Yokohama 226-8503, Japan. Correspondence and requests for materials should be addressed to H.D. (e-mail: hdaicho@koito.co.jp).
} 
$\mathrm{R}$ ecently, white light-emitting diodes (LEDs) have been receiving attention as light sources because of their energy efficiency ${ }^{1,2}$. The major white LEDs presently in the market are phosphor-converted LEDs (pc-LEDs) made of a blue InGaN chip and a yellow phosphor, $(\mathrm{Y}, \mathrm{Gd})_{3}(\mathrm{Al}, \mathrm{Ga})_{5} \mathrm{O}_{12}: \mathrm{Ce}^{3+}$ (abbreviated YAG: $\left.\mathrm{Ce}^{3+}\right)^{3}$. Its luminescence efficiency exceeds that of fluorescent lamps ${ }^{4,5}$. However, as pc-LEDs have much smaller emission areas than conventional light sources, their luminance rises excessively with increasing luminous efficiency, and consequently they produce an uncomfortable glare ${ }^{6}$. The emission areas of pc-LEDs are small, because the yellow-emitting phosphor must be located close to the blue chip to produce a uniform hue; therefore, the phosphor layer is typically deposited on the blue chip so that the chip and the layer have the same geometric area.

There have been many reports describing the development of phosphors ${ }^{7-16}$ for violet chips, which have an external quantum efficiency that is intrinsically higher than that of blue chips ${ }^{17-19}$. A pc-LED with a violet chip generates white light only from the luminescence of the phosphors. However, none of the previous reports dealt with glare suppression, which is a major obstacle to the practical application of pc-LEDs. In a pc-LED with a violet chip, two or more phosphors must be mounted on the chip. A combination of blue and yellow phosphors, or a combination of blue, green and red phosphors, is commonly used in pc-LEDs. Glare can be suppressed by expanding the emission area, but such expansion leads to a non-uniform hue. As the green, yellow and red phosphors emit undesirable luminescence by absorbing the light emitted from the blue phosphor ${ }^{20}$, therefore, phosphors that are not excited by the blue light are required. We approached this problem by tuning the excitation properties of the phosphor.

In this article, we report a new phosphor, $\left(\mathrm{Ca}_{1-x-y}, \mathrm{Sr}_{x}, \mathrm{Eu}_{y}\right)_{7}$ $\left(\mathrm{SiO}_{3}\right)_{6} \mathrm{Cl}_{2}$ (abbreviated Cl_MS:Eu ${ }^{2+}$ ), which emits yellow luminescence upon violet excitation, with an internal quantum efficiency (IQE) higher than $90 \%$ and very weak absorption in the blue wavelength region. These properties allowed us to fabricate a pc-LED composed of a violet chip combined with $\mathrm{Cl} \_\mathrm{MS}: \mathrm{Eu}^{2+}$ and a blue phosphor, $(\mathrm{Ca}, \mathrm{Sr})_{5}\left(\mathrm{PO}_{4}\right)_{3} \mathrm{Cl}: \mathrm{Eu}^{2+}\left(\mathrm{APT}: \mathrm{Eu}^{2+}\right)^{21}$. This pc-LED had luminous flux, colour temperature and colour-rendering index values that were adequate for practical use, and it emitted white light with a uniform hue and suppressed glare, thus satisfying the requirements for general illumination. The host, Cl_MS, is a new material with a unique layered structure discovered in this work.

\section{Results}

Search for a phosphor material. In our search for an appropriate phosphor, we explored chemically stable oxide-based materials activated with $\mathrm{Eu}^{2+}$, which allows $5 d-4 f$ transitions. We considered the following features when designing the host material. The primary feature was a mixed-ligand system. In most $\mathrm{Eu}^{2+}$ oxides, $\mathrm{Eu}^{2+}$ absorbs in the ultraviolet wavelength region. Shifting the absorption band to the violet region requires a relatively large crystal-field splitting. A strong crystal field is generated when $\mathrm{Eu}^{2+}$ is located at a site with a distorted co-ordination field ${ }^{22}$, and such a site can be created with a mixed-ligand system. Here we chose oxide and chloride ions as the ligands. The second feature was a layered structure. To obtain a chemically stable phosphor, we chose a host with a layered structure of hydrophilic metal chloride layers alternating with oxide layers. We expected the layering to stabilize the chemically unstable metal chloride layer. As phosphors with layered structures often suffer from substantial thermal quenching of luminescence owing to loose interlayer bonding 23,24 , we explored materials that exhibited a small degree of thermal quenching.

A survey of materials conducted with these features in mind led us to focus on chlorosilicates ${ }^{25-27}$ and ultimately to design a new phosphor material, $\mathrm{Cl} \_\mathrm{MS}: \mathrm{Eu}^{2+}$.
Crystal structures of Cl_MS and Cl_MS:Eu ${ }^{2+}$. We determined the crystal structures of an undoped Cl_MS single crystal and several $\mathrm{Eu}^{2+}$-doped powder samples by using synchrotron radiation X-ray diffraction (SR-XRD) at the SPring-8 synchrotron facility in Japan (see Supplementary Methods and Supplementary Tables S1 and S2). The structure of Cl_MS: $\mathrm{Eu}^{2+}$ was found to consist of alternating metal silicate and metal chloride layers along the $\mathrm{c}$ axis of a monoclinic unit cell (Fig. 1a). The chemical composition of Cl_MS: $\mathrm{Eu}^{2+}$ was determined to be $\left(\mathrm{Ca}_{1-x-y}, \mathrm{Sr}_{x}, \mathrm{Eu}_{y}\right)_{7}\left(\mathrm{SiO}_{3}\right)_{6} \mathrm{Cl}_{2}(0.167$ $<x /(1-x-y)<2.5 ; 0.01<y<0.3)$. Cl_MS:Eu ${ }^{2+}$ had three cationic sites referred to as $M(1), M(2)$ and $M(3)$, which could accommodate $\mathrm{Ca}, \mathrm{Sr}$ or Eu. The M(1) site in the metal silicate layer was occupied only by $\mathrm{Ca}$ and was co-ordinated by six oxide ions of $\mathrm{Si}_{3} \mathrm{O}_{9}$ units ${ }^{28}$ to form a $\left[\mathrm{Ca}\left(\mathrm{SiO}_{3}\right)_{6}\right]$ framework (see Supplementary Fig. S1). The $\mathrm{M}(2)$ site was in the metal chloride layer and was occupied by a $\mathrm{Sr}$ or Eu ion. This site was co-ordinated by a chloride ion in the metal chloride layer and by seven oxide ions, and thus the site bridged the two metal silicate layers (Fig. 1b). The M(3) site at the boundary between the metal silicate and metal chloride layers was occupied by a $\mathrm{Ca}, \mathrm{Sr}$ or Eu ion, and was co-ordinated by five oxygen ions in the metal silicate layer and by two chloride ions in the metal chloride layer (Fig. 1b). We have provided crystal information files in the Supplementary Data Sets 1 and 2. Supplementary Data Set 1 includes the crystal structures of an undoped single crystal of Cl_MS at 100 and $300 \mathrm{~K}$. Supplementary Data Set 2 includes the crystal structures of Cl_MS: $\mathrm{Eu}^{2+}$ at 100 and $300 \mathrm{~K}$.

Luminescence spectrum of $\mathbf{C l} \_\mathbf{M S}: \mathrm{Eu}^{2+}$. The luminescence spectrum of $\left(\mathrm{Ca}_{0.37} \mathrm{Sr}_{0.53} \mathrm{Eu}_{0.10}\right)_{7}\left(\mathrm{SiO}_{3}\right)_{6} \mathrm{Cl}_{2}$ obtained with a $400-\mathrm{nm}$ excitation at room temperature exhibited a single band at $580 \mathrm{~nm}$, with a full width at half-maximum of $3.6 \times 10^{3} \mathrm{~cm}^{-1}$ (Fig. 2a). The corresponding excitation spectrum exhibited strong absorption at wavelengths of $410 \mathrm{~nm}$ or shorter, but the intensity of the absorption rapidly decreased at wavelengths longer than $420 \mathrm{~nm}$. Consequently, an $~ 450$-nm blue light was only weakly absorbed by Cl_MS:Eu ${ }^{2+}$. Thus, the excitation spectrum of Cl_MS: $\mathrm{Eu}^{2+}$ distinctly differed from the excitation spectra of other phosphors used for violet excitation. For example, the excitation spectra of $(\mathrm{Ba}, \mathrm{Sr})_{2} \mathrm{SiO}_{4}: \mathrm{Eu}^{2+}$ (ref. 29) and (Sr,Ca)AlSiN ${ }_{3}: \mathrm{Eu}^{2+}$ (refs 30,31) exhibit considerable absorption intensity around $450 \mathrm{~nm}$ (Fig. 2a). In addition, the magnitude of the Stokes shift $(\Delta S)$ for Cl_MS:Eu ${ }^{2+}$ was $6.9 \times 10^{3} \mathrm{~cm}^{-1}$, which was much larger than that observed for conventional phosphors (Fig. 2a). The emission colour of Cl_MS:Eu ${ }^{2+}$ could be tuned by varying the $\mathrm{Ca} / \mathrm{Sr}$ atomic ratio. We examined the $\mathrm{Ca}$ fraction dependence of the dominant wavelength (that is, the wavelength of monochromatic light that was the same colour as the band luminescence), and found that as the $\mathrm{Ca} / \mathrm{Sr}$ ratio was increased the dominant wavelength was linearly red-shifted (Fig. 2b). The IQEs and absorptions at the dominant wavelength were almost equivalent. The luminescence spectra of typical samples are shown in Supplementary Fig. S2. The luminescence intensity of Cl_MS: $\mathrm{Eu}^{2+}$ was stable to $\mathrm{Eu}^{2+}$ concentration and temperature. Even though the $\mathrm{Eu}^{2+}$ concentration in Cl_MS: $\mathrm{Eu}^{2+}$ increased by more than 20 atom\%, the samples consisted almost completely of a single phase that contained only a few impurities (see Supplementary Fig. S3). No concentration quenching occurred at $\mathrm{Eu}^{2+}$ concentrations up to 20 atom\% (Fig. $2 \mathrm{c}$ and Supplementary Table S3). The highest luminescence intensity was observed at a dopant level of 10 atom\%, $\left(\mathrm{Ca}_{0.37} \mathrm{Sr}_{0.53} \mathrm{Eu}_{0.10}\right)_{7}\left(\mathrm{SiO}_{3}\right)_{6}$ $\mathrm{Cl}_{2}$, and the IQE and the absorption were $94 \%$ and $90 \%$, respectively, upon excitation by a 407-nm laser diode. A luminescence intensity of $75 \%$ relative to the room temperature value was observed at $423 \mathrm{~K}$ (Fig. 2d). This temperature dependence was comparable to that of YAG: $\mathrm{Ce}^{3+}$. A life test in air at $85 \%$ humidity and $358 \mathrm{~K}$ showed that the variation in luminescence intensity was within $2 \%$ after $2,000 \mathrm{~h}$; this variation was less than the variation observed for $(\mathrm{Ba}, \mathrm{Sr})_{2} \mathrm{SiO}_{4}: \mathrm{Eu}^{2+}$ after only $500 \mathrm{~h}$ of testing (Fig. 2e). 

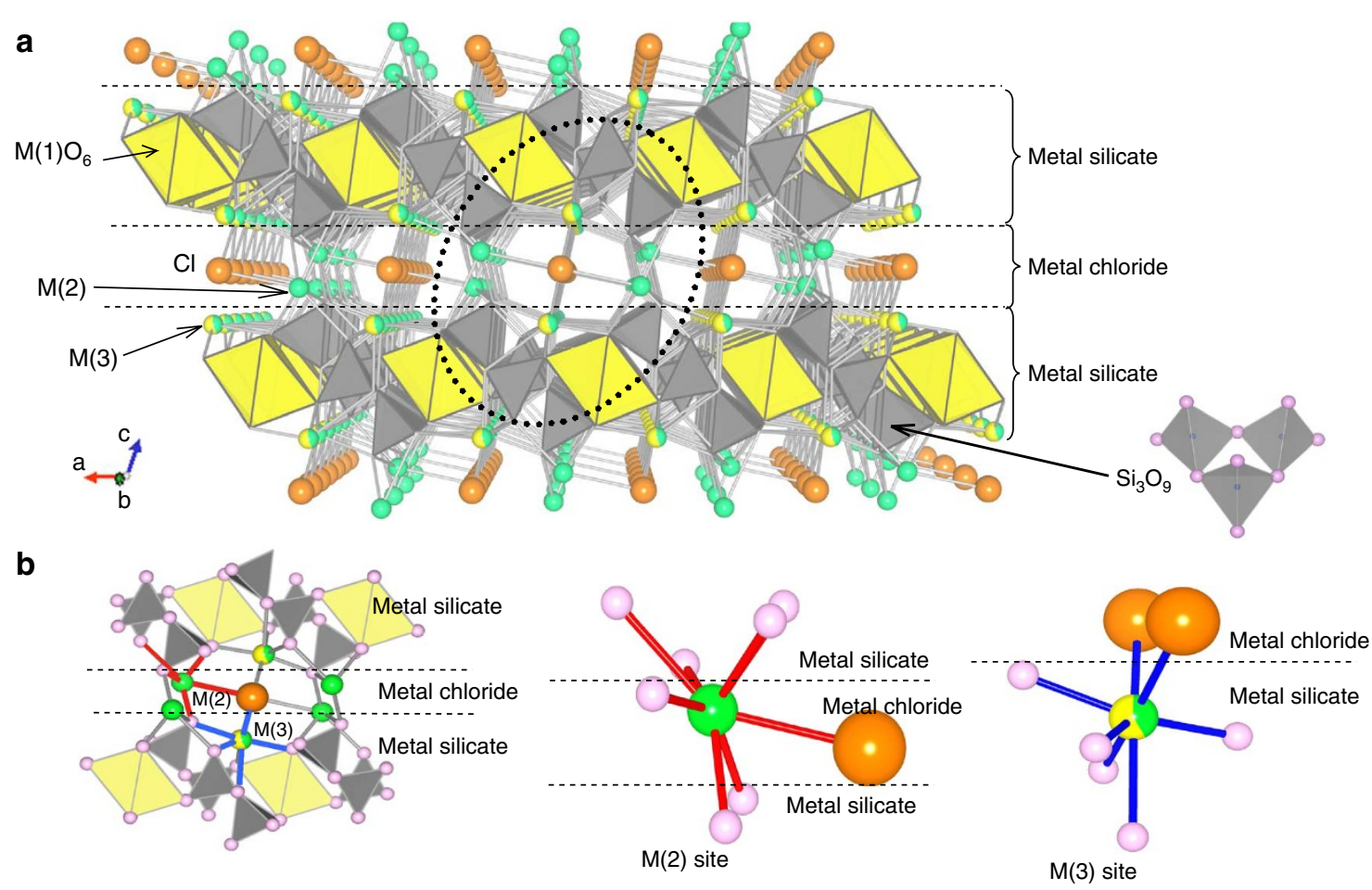

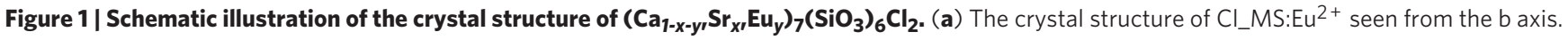
Grey tetrahedra represent $\mathrm{SiO}_{4}$ units, yellow octahedra represent $\mathrm{M}(1) \mathrm{O}_{6}$ units, orange spheres represent chloride ions, green spheres represent $\mathrm{M}(2)$ ions, and yellow and green spheres represent M(3) ions. The horizontal dashed lines indicate boundaries between the metal silicate and metal chloride layers. The area indicated by the bold dotted circle is shown in detail in $\mathbf{b}$. The geometry of a $\mathrm{Si}_{3} \mathrm{O}_{9}$ unit is shown at the lower right of this panel. (b) Detailed illustration of the chemical bonds around the $M(2)$ and $M(3)$ sites. The red and blue sticks indicate chemical bonds between anions and the $M(2)$ and $M(3)$ sites, respectively. Small pink spheres represent oxide ions. The coordination geometries of the $M(2)$ and $M(3)$ sites are also shown separately.

$\mathrm{Eu}^{2+}$ sites of $\mathbf{C l} \_\mathbf{M S}: \mathbf{E u}^{2+}$. Upon excitation with ultraviolet radiation (Fig. 3a), a peak at $480 \mathrm{~nm}$ appeared in the luminescence spectrum of $\left(\mathrm{Ca}_{0.40} \mathrm{Sr}_{0.56} \mathrm{Eu}_{0.04}\right)_{7}\left(\mathrm{SiO}_{3}\right)_{6} \mathrm{Cl}_{2}$. The excitation spectrum of this emission band $(480 \mathrm{~nm})$ differed from that monitored at $580 \mathrm{~nm}$; the former spectrum exhibited a narrow band peaking at $310 \mathrm{~nm}$ with weak violet absorption. This result confirmed that Cl_MS:Eu ${ }^{2+}$ had two different $\mathrm{Eu}^{2+}$-occupied sites, the $\mathrm{M}(2)$ and $\mathrm{M}(3)$ sites (Fig. 1b). To clarify the correspondence between the two crystallographic sites and the emission bands, we investigated the temperature dependence of the luminescence intensity and of the crystal structure. We plotted the radiative decay curves for $\left(\mathrm{Ca}_{0.40} \mathrm{Sr}_{0.56} \mathrm{Eu}_{0.04}\right)_{7}\left(\mathrm{SiO}_{3}\right)_{6} \mathrm{Cl}_{2}$ at 100 and $300 \mathrm{~K}$ upon excitation with a 266-nm laser pulse with monitoring at 480 and $580 \mathrm{~nm}$ (Fig. 3b,c). All the decay curves consisted of fast and slow components (with lifetimes $\tau_{1}$ and $\tau_{2}$, respectively), and were fitted by double exponential components. Here we discuss the fast component of each decay curve. The shorter lifetime of the 480-nm band $\left(\tau_{1}\right)$ showed large temperature dependence between 100 and $300 \mathrm{~K}$, whereas neither $\tau_{1}$ nor $\tau_{2}$ of the 580 -nm band changed substantially with temperature. The SR-XRD analysis (Fig. $3 \mathrm{~d}$ ) revealed that the local structure of the $\mathrm{M}(2)$ site changed markedly with increasing temperature; three of the seven $\mathrm{M}(2)-\mathrm{O}$ bonds lengthened by more than $0.02 \AA$. In contrast, the bond lengths at the $\mathrm{M}(3)$ site did not change markedly with increasing temperature (Fig. $3 \mathrm{~d})$. Consequently, we attributed the $580-\mathrm{nm}$ band to $\mathrm{Eu}^{2+}$ at the $\mathrm{M}(3)$ site and the $480-\mathrm{nm}$ band to $\mathrm{Eu}^{2+}$ at the $\mathrm{M}(2)$ site.

\section{Discussion}

The $\mathrm{M}(3)$-site $\mathrm{Eu}^{2+}$ ion, which gave rise to the $580-\mathrm{nm}$ band, was co-ordinated by the mixed ligand, that is, a single bridging oxygen ion, four non-bridging oxygen ions and two chloride ions (Fig. 1b), and was located at the boundary between the metal silicate and metal chloride layers. The asymmetric coordination structure of the $\mathrm{M}(3)$ site resulted in a large crystal-field splitting and an absorption band at a wavelength in the violet region, but not extending to the blue region. We evaluated the asymmetry of the $\mathrm{Eu}^{2+}$ sites by calculating electrical field gradients (EFGs) for the $\mathrm{M}(2)$ and $\mathrm{M}(3)$ sites using density functional theory (DFT). The initial structural parameters were taken from single-crystal SR-XRD data for $\mathrm{CaS}$ $\mathrm{r}_{2}\left(\mathrm{Ca}_{0.55} \mathrm{Sr}_{0.45}\right)_{4} \mathrm{Si}_{6} \mathrm{O}_{18} \mathrm{Cl}_{2}$ at $100 \mathrm{~K}$. In the calculation, we modelled the mixed occupation of the $\mathrm{M}(3)$ sites with $\mathrm{Ca}$ and $\mathrm{Sr}$ by the ordered structure shown in Supplementary Fig. S4. To obtain a relaxed structure, we optimized the geometry with the Vienna Ab Initio Simulation Package code ${ }^{32}$ using the projected, augmented plane-wave method ${ }^{33}$. The EFG parameters were calculated with the WIEN2k programme package ${ }^{34}$, which adopts a full-potential, all-electron method that utilizes the full-potential, linearized, augmented plane-wave + local orbitals approach ${ }^{35}$. In each calculation procedure, the exchange and correlation potentials within the DFT were calculated with the Perdew-Burke-Ernzerhof generalized gradient approximation ${ }^{36}$. The principal axes of the EFG tensors of the $\mathrm{Sr}^{2+}$ and $\mathrm{Ca}^{2+}$ ions at the $\mathrm{M}(2)$ and $\mathrm{M}(3)$ sites, respectively, are illustrated schematically in Fig. 4a, and the EFG tensor elements at the $\mathrm{M}(2)$ and $\mathrm{M}(3)$ sites calculated by DFT are summarized in Table 1 . The asymmetry parameters $\eta$ of the $\mathrm{M}(2)$ and $\mathrm{M}(3)$ sites could be derived by using the EFG tensor elements (defined at the bottom of Table 1 ). The value of $\eta$ at the M(3) site was clearly much higher than that at the $\mathrm{M}(2)$ site.

The changes in the local structure of the $\mathrm{M}(3)$ site due to the increase in the $\mathrm{Ca}$ fraction induced a red shift of the luminescence. 

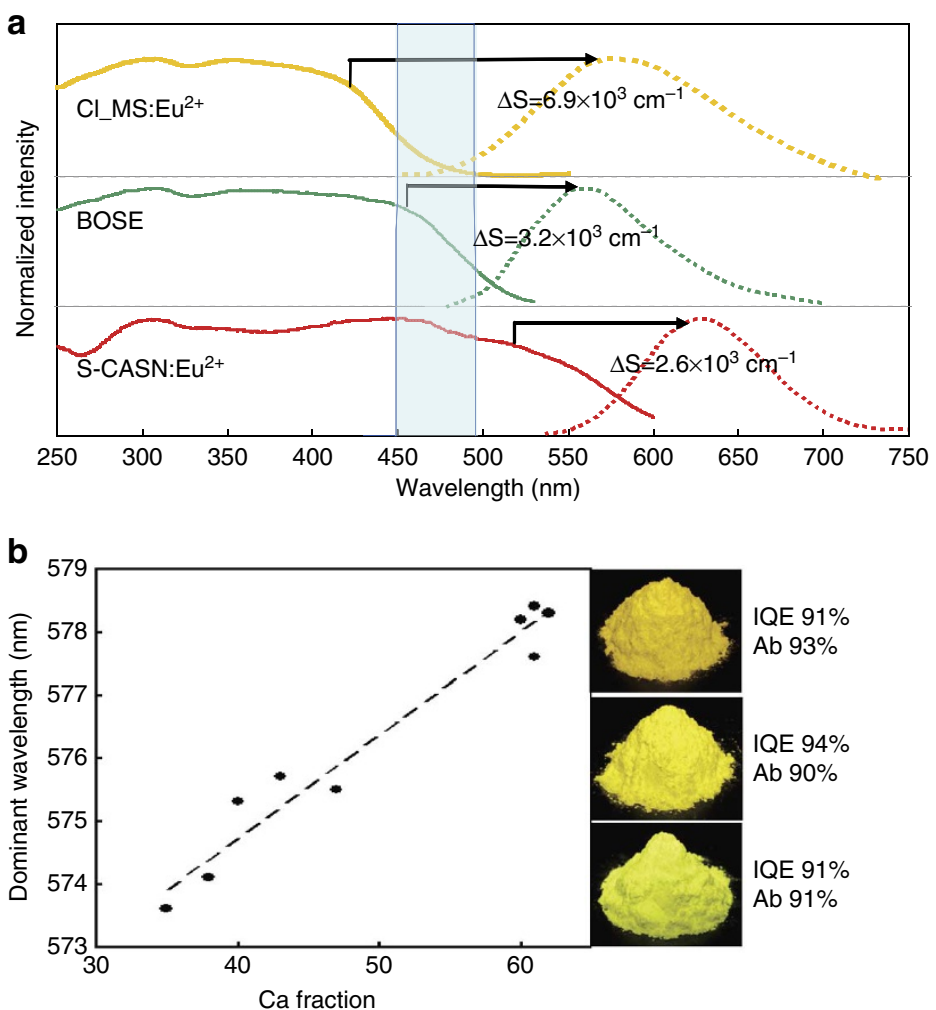
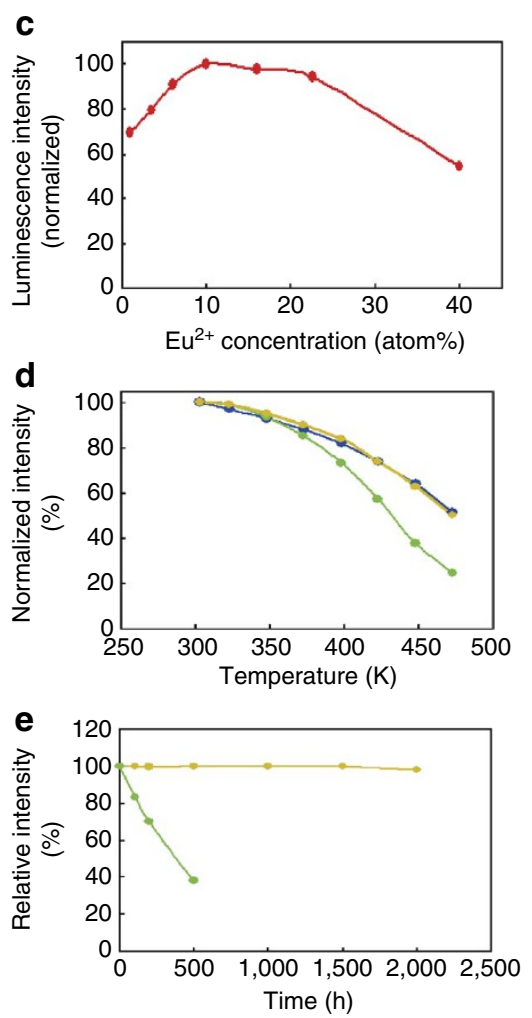

Figure 2 | Luminescence properties of CI_MS:Eu²+ . (a) Luminescence (dotted curves) and excitation spectra (solid curves) of phosphors with excitation at $400 \mathrm{~nm}$ and monitoring at $580 \mathrm{~nm}$. The yellow curves show spectra of $\left(\mathrm{Ca}_{0.37} \mathrm{Sr}_{0.53} \mathrm{Eu}_{0.10}\right)_{7}\left(\mathrm{SiO}_{3}\right)_{6} \mathrm{Cl}_{2}$. For comparison, the spectra of two conventional phosphors are also shown; $\left(\mathrm{Ba}, \mathrm{Sr}_{2} \mathrm{SiO}_{4}: \mathrm{Eu}^{2+}(\mathrm{BOSE}\right.$; green curves; excitation at $400 \mathrm{~nm}$; monitoring at $560 \mathrm{~nm})$ and $\left(\mathrm{Sr}_{1} \mathrm{Ca}^{2}\right) \mathrm{AlSiN}_{3}: \mathrm{Eu}^{2+}\left(\mathrm{S}-\mathrm{CASN}: \mathrm{Eu}^{2+}\right.$; red curves; excitation at $400 \mathrm{~nm}$; monitoring at $630 \mathrm{~nm}$ ). The area hatched in blue indicates the wavelengths of blue light. The Stokes shift values $(\triangle S)$ are also shown. (b) Dominant wavelength of luminescence as a function of $\mathrm{Ca}$ fraction in $\mathrm{Cl} \_\mathrm{MS}: \mathrm{Eu}^{2+}$. The IQE and absorption (Ab) values for Cl_MS:Eu ${ }^{2+}$ samples with luminescence at various dominant wavelengths are also shown. (c) Normalized luminescence intensity for the 400-nm excitation of Cl_MS: $\mathrm{Eu}^{2+}$ as a function of $\mathrm{Eu}^{2+}$ concentration. (d) Temperature dependence of luminescence intensity normalized to intensity at $303 \mathrm{~K}$. The yellow, blue and green curves indicate $\mathrm{Cl} \_\mathrm{MS}: \mathrm{Eu}^{2+}, \mathrm{YAG}: \mathrm{Ce}^{3+}$ and $\mathrm{BOSE}$, respectively. (e) Relative luminescence intensity as a function of time at $358 \mathrm{~K}$ and $85 \%$ humidity. The yellow and green curves indicate $\mathrm{Cl} \_\mathrm{MS}: \mathrm{Eu}^{2+}$ and BOSE, respectively.

Anisotropic lattice contraction occurred along the a axis (see Supplementary Fig. S5), owing to shortening of the $\mathrm{O}(5)-\mathrm{M}(3)-\mathrm{O}(6)$ bonds with increasing $\mathrm{Ca}$ fraction (Fig. 4b). This contraction induced substantial deformation around the $\mathrm{M}(3)$ site. As such deformation widened the crystal-field splitting, the 580-nm luminescence band was red-shifted by a reduction of the lowest $5 d$ energy level. These results are consistent with the calculated results, indicating that the $\eta$ value for $\mathrm{Ca}$ at the $\mathrm{M}(3)$ site was larger than that for Sr (Table 1).

Cl_MS:Eu ${ }^{2+}$ exhibited a high IQE. IQE is given by Yamamoto ${ }^{37}$

$$
\mathrm{IQE}=W /(W+N)
$$

where $W$ is the luminescence radiation probability and $N$ is the non-radiative transition probability. The main factors that affect the non-radiative transition probability are thermal quenching and concentration quenching. The IQE of $\mathrm{Cl} \_\mathrm{MS}: \mathrm{Eu}^{2+}$ was very high because the influence of these factors was small owing to the following reasons.

Thermal expansion of Cl_MS:Eu ${ }^{2+}$ was anisotropic. The lattice constants increased linearly with increasing temperature from 100 to $573 \mathrm{~K}$ as shown in Supplementary Fig. S6. The linear thermal expansion co-efficient along the $\mathrm{C}$ axis, the direction perpendicular to the layers, was larger than that along the $\mathrm{a}-\mathrm{and} \mathrm{b}$ axes. The values along the $c^{-}, \mathrm{a}-$ and $\mathrm{b}$ axes at $300 \mathrm{~K}$ were $1.8 \times 10^{5}, 5.4 \times 10^{6}$ and $7.5 \times 10^{6} \mathrm{~K}^{-1}$, respectively. At the $\mathrm{M}(2)$ site, which bridged the metal silicate layers, the cation-oxygen bond lengths were markedly increased by thermal expansion of the interlayer separation. These lengthened bonds resulted in a shorter luminescence lifetime for the 480 -nm band. In contrast, the change in the $\mathrm{Eu}^{2+}$-anion distance was less at the $\mathrm{M}(3)$ site, which had few co-ordinated oxide ions in the stacked-layer direction. Consequently, thermal quenching of the $580-\mathrm{nm}$ band was sufficiently small for practical applications, despite the material's layered structure.

Concentration quenching of the main emission band (the 580-nm band) of Cl_MS:Eu ${ }^{2+}$ was not observed (Fig. 2c), but the $480-\mathrm{nm}$ band was drastically affected by concentration quenching (see Supplementary Fig. S7). We cannot explain, on the basis of the experimental results, why concentration quenching of the $\mathrm{M}(3)$ site did not occur. The conspicuous difference in the sensitivity of the $\mathrm{M}(2)$ and $\mathrm{M}(3)$ sites to concentration quenching appears to have to do with local asymmetry around each site, which controlled the energy transfer processes from the excited $\mathrm{Eu}^{2+}$. There have been reports indicating that phosphors with a low-dimensional arrangement of the dopant ions show little concentration quenching ${ }^{38}$. In such phosphors, the stoichiometric phosphors (100\% dopant ion) are also contained ${ }^{39,40}$. The dopant ion site of these phosphors has higher asymmetry, owing to low-dimensional arrangement. This observation is consistent with the fact that the $\mathrm{M}(3)$ site, which had lower symmetry and a low-dimensional arrangement, did not exhibit concentration quenching. 
a

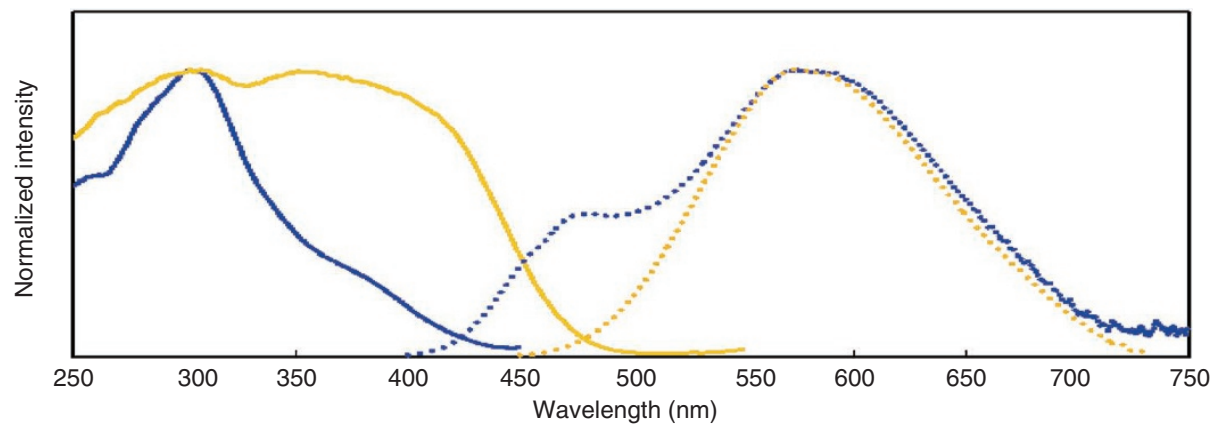

b

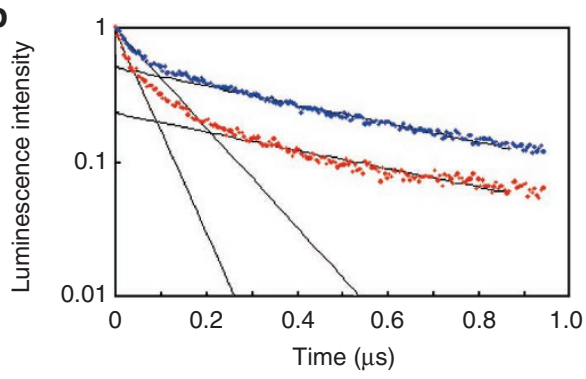

c

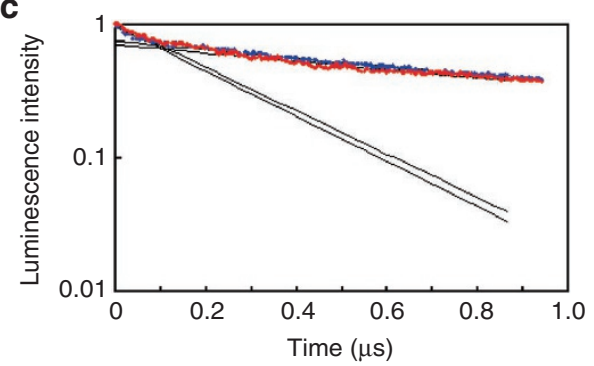

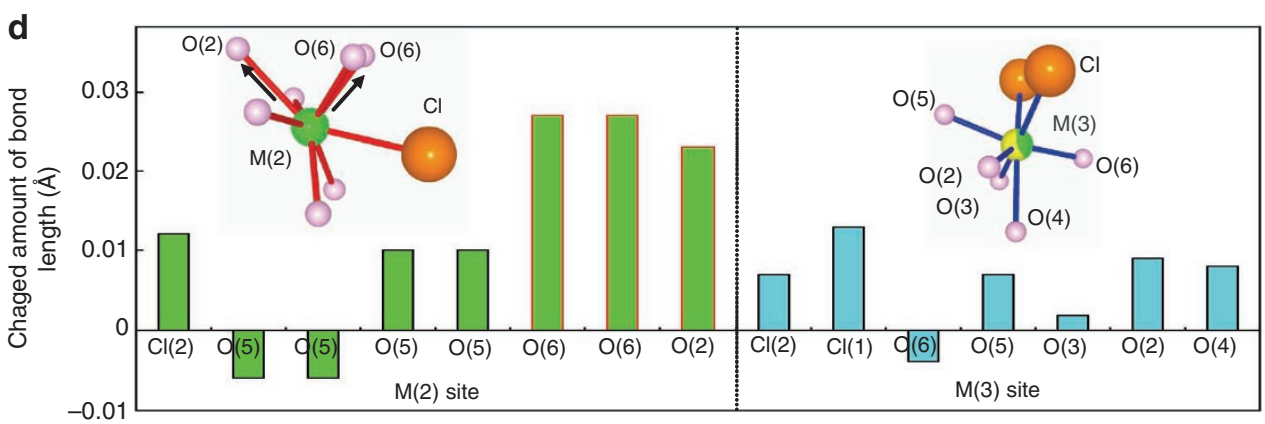

Figure 3 | Temperature dependence of the luminescence properties and bond lengths of $\left(\mathrm{Ca}_{0.40} \mathbf{S r}_{0.56} \mathrm{Eu}_{0.04}\right)_{7}\left(\mathrm{SiO}_{3}\right)_{6} \mathrm{Cl}_{2}$. (a) Room temperature luminescence spectra with excitation at 310 (dotted blue curve) and $400 \mathrm{~nm}$ (dotted yellow curve), and excitation spectra monitored at 480 (solid blue curve) and $580 \mathrm{~nm}$ (solid yellow curve). (b) Radiative decay curves of $\mathrm{Eu}^{2+}$ luminescence at $480 \mathrm{~nm}$. Blue and red lines indicate data measured at 100 and $300 \mathrm{~K}$, respectively. Both decay curves consisted of fast and slow components. The lifetime $\left(\tau_{1}\right)$ of the fast component was $0.12 \mu \mathrm{s}$ at $100 \mathrm{~K}$ and $0.06 \mu \mathrm{s}$ at $300 \mathrm{~K}$. The lifetime $\left(\tau_{2}\right)$ of the slow component was $0.62 \mu \mathrm{s}$ at $100 \mathrm{~K}$ and $0.63 \mu \mathrm{s}$ at $300 \mathrm{~K}$. (c) Radiative decay curves of Eu ${ }^{2+}$ luminescence at $580 \mathrm{~nm}$. Blue and red lines indicate data measured at 100 and $300 \mathrm{~K}$, respectively. Both decay curves consisted of fast and slow components. The lifetime $\left(\tau_{1}\right)$ of the fast component was $0.26 \mu \mathrm{s}$ at $100 \mathrm{~K}$ and $0.27 \mu \mathrm{s}$ at $300 \mathrm{~K}$. The lifetime $\left(\tau_{2}\right)$ of the slow component was $1.4 \mu \mathrm{s}$ at $100 \mathrm{~K}$ and $1.4 \mu \mathrm{s}$ at $300 \mathrm{~K}$.

(d) Difference of the bond distances at 100 and $300 \mathrm{~K}$ for the $\mathrm{M}(2)$ and $\mathrm{M}(3)$ sites, as indicated by SR-XRD data. Positive values indicate bond elongation, and negative values indicate bond shortening with increasing temperature.

To evaluate the practical applicability of the $\mathrm{Cl} \_\mathrm{MS}: \mathrm{Eu}^{2+}$ phosphor, we fabricated three sample pc-LEDs consisting of a violet or blue chip encapsulated in translucent resin containing various phosphors (Fig. 5a). Sample 1 was prepared with a violet chip coupled with $\left(\mathrm{Ca}_{0.37} \mathrm{Sr}_{0.53} \mathrm{Eu}_{0.10}\right)_{7}\left(\mathrm{SiO}_{3}\right)_{6} \mathrm{Cl}_{2}$ and the blue phosphor APT: $\mathrm{Eu}^{2+}$ (Fig. 5b). For reference, we also prepared pc-LEDs of the same structure with a blue chip coupled with YAG:Ce ${ }^{3+}$ (sample 2, Fig. 5c) and with a violet chip coupled with three primary-colour phosphors (sample 3, Fig. 5d). The differences in the emission colour distribution in the emission area of these three samples were measured with a two-dimensional colour analyser (Fig. 5e,f,g). Sample 1 exhibited a uniform hue over the entire resin dome, similar to that of an incandescent lamp, but samples 2 and 3 showed red-shifted colour on the rim of the dome, resulting in a non-uniform hue. The performance of these three samples is summarized in Supplementary Table S4.

Sample 1 exhibited a luminous flux of $88 \mathrm{~lm}$ and a luminance of $1.0 \mathrm{~cd} \mathrm{~mm}^{-2}$ at an operating current of $350 \mathrm{~mA}$. For comparison, a surface-mount device (SMD) pc-LED, which is a commonly used type of pc-LED, exhibited a luminous flux of $85 \mathrm{~lm}$ and a luminance of $11 \mathrm{~cd} \mathrm{~mm}^{-2}$. The SMD pc-LED consisted of a thin phosphor layer deposited on a chip mounted at the bottom of a small casing (see Supplementary Fig. S8). The unified glare rating (UGR), which is used to evaluate glare, is given by the CIE ${ }^{41}$

$$
\mathrm{UGR}=8 \log \left[0.25 / L b \cdot \Sigma\left(L^{2} \omega / p^{2}\right)\right]
$$

where $L_{\mathrm{b}}$ is the background luminance $\left(\mathrm{cd} \mathrm{m}^{-2}\right), L$ is the source luminance $\left(\mathrm{cd} \mathrm{m}^{-2}\right), \omega$ is the solid angle of the source (sr) and $p$ is the position index (the positional relationship between the light source and the line of sight). The form of this equation indicates that as the source luminance increases, UGR rises and glare becomes uncomfortable. In sample 1, the luminance was reduced to less than $1 / 10$ of that for the SMD pc-LED without a decrease in luminous flux; thus, unwanted glare was suppressed (see Supplementary Methods and Supplementary Fig. S9).

After continuous operation at $350 \mathrm{~mA}$ for $3,000 \mathrm{~h}$, the luminous flux of sample 1 was still at least $96 \%$ of its initial value. The colour temperature was $4,100 \mathrm{~K}$ for sample 1 , and the temperature 
could be tuned from 3,010 to $5,070 \mathrm{~K}$ by varying the $\mathrm{Ca}$ fraction (Fig. 5h).

We also fabricated a line-shaped module consisting of a row of pc-LEDs fabricated with Cl_MS:Eu ${ }^{2+}$ (Fig 5i,j). The module contained 18 violet chips aligned with a pitch of $8 \mathrm{~mm}$. The chips were encapsulated thickly in a semi-cylindrical form, with resin containing Cl_MS: $\mathrm{Eu}^{2+}$ phosphors. White-light emission from this device was uniform in hue over an area of $150 \times 8 \mathrm{~mm}^{2}$, and the module exhibited a luminous flux of $500 \mathrm{~lm}$ with a luminous efficiency of $90 \operatorname{lm~} \mathrm{W}^{-1}$.

In conclusion, we have developed a phosphor for a violet chip, $\mathrm{Cl} \_\mathrm{MS}: \mathrm{Eu}^{2+}$, based on a newly discovered host crystal with a mixed-ligand system and a layered structure. Cl_MS:Eu ${ }^{2+}$ exhibited a large Stokes shift, efficiently converting violet light to yellow luminescence, and the luminous efficiency showed good temperature dependence despite the material's layered structure. The use of Cl_MS:Eu ${ }^{2+}$ in conjunction with violet LED chips allowed us to fabricate glareless pc-LEDs suitable for general illumination with uniform hue, moderate luminance, adequate colour-rendering index

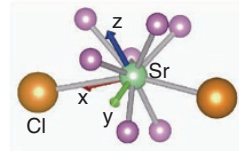

$M(2)$ site

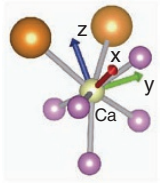

$M(3)$ site

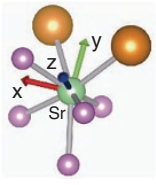

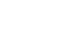

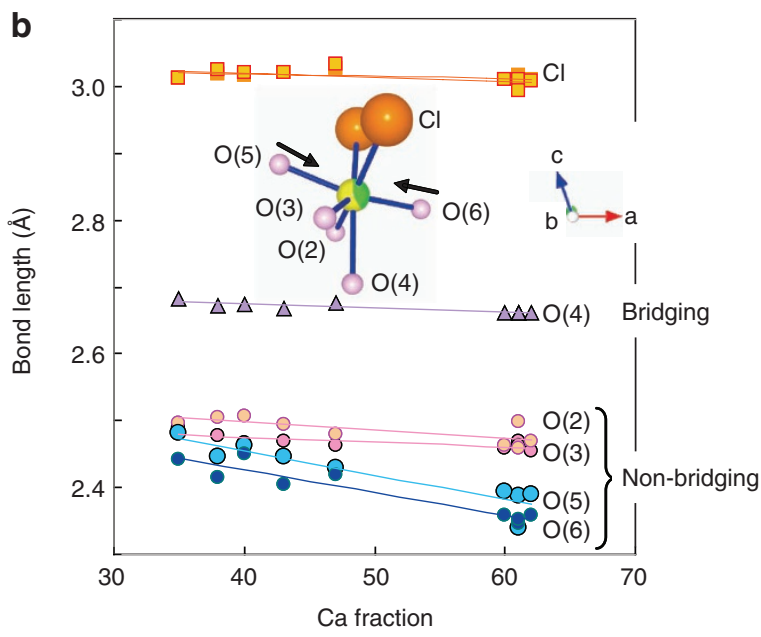

Figure 4 | Local structure of $\mathrm{Eu}^{2+}$ occupancy sites of $\mathrm{Cl} \_\mathrm{MS}: \mathrm{Eu}^{2+}$. (a) Schematic illustrations of principal axes of EFG tensors of $\mathrm{Sr}^{2+}$ and $\mathrm{Ca}^{2+}$ ions at the $\mathrm{M}(2)$ and $\mathrm{M}(3)$ sites. (b) Effect of Ca fraction on $M(3)$-anion bond distance at the $M(3)$ site, as indicated by SR-XRD data: $\mathrm{M}(3)-\mathrm{Cl}$ (orange squares); $\mathrm{M}(3)-\mathrm{O}(4)$ (violet triangles; $\mathrm{O}(4)$ is a bridging oxygen); and $\mathrm{M}(3)-\mathrm{O}(2),-\mathrm{O}(3),-\mathrm{O}(5)$ and $-\mathrm{O}(6)$ (pink and blue circles; these are non-bridging oxygens). and high luminous flux. Furthermore, pc-LEDs prepared with Cl_MS:Eu ${ }^{2+}$ can be expected to permit the fabrication of light sources with new shapes, such as domes or elongated semicylinders.

\section{Methods}

Sample preparation. Single crystals of undoped Cl_MS were grown by the selfflux method, in which $\mathrm{SrCl}_{2}$ acted as both a starting material and a flux. For the formation of a sufficient quantity of liquid flux, $\mathrm{SrCl}_{2}$ was added in excess (7.5 times the stoichiometric amount). The other starting material, $\mathrm{SiO}_{2}$, was also added in excess ( 1.2 times the stoichiometric amount) because it is not very reactive. $\mathrm{SrCl}_{2}, \mathrm{SiO}_{2}$ and a stoichiometric amount of $\mathrm{CaCO}_{3}$, all of $3 \mathrm{~N}$ or higher purity, were thoroughly mixed and pelletized. The pellets were heated at $1,030^{\circ} \mathrm{C}$ for $36 \mathrm{~h}$ in air and then cooled to room temperature at a rate of $300^{\circ} \mathrm{Ch}^{-1}$. The resulting Cl_MS crystals were collected by washing off the remaining flux with warm water.

We prepared samples of $\mathrm{Eu}^{2+}$-doped Cl_MS by using the method described for Cl_MS, with $\mathrm{Eu}_{2} \mathrm{O}_{3}$ or $\mathrm{EuCl}_{3}$ as the Eu source. The $\mathrm{Eu}^{2+}$ fraction per divalent metal ion was $3-50$ atom\%. The pellets were fired at $1,030^{\circ} \mathrm{C}$ for $10 \mathrm{~h}$ in a reducing atmosphere of $\mathrm{H}_{2} / \mathrm{N}_{2}(5 / 95)$.

Fabrication of pc-LEDs. Phosphors of different emission colours were mixed at the following weight ratios to provide white emission of specified colour coordinates: Cl_MS:Eu ${ }^{2+} / \mathrm{APT}: \mathrm{Eu}^{2+}=1.8 / 1.0$ for pc-LED sample 1, 100\% YAG:Ce ${ }^{3+}$ for pc-LED sample 2 and $(\mathrm{Sr}, \mathrm{Ca}) \mathrm{AlSiN}_{3}: \mathrm{Eu}^{2+} / \beta-\mathrm{SiAlON} / \mathrm{APT}: \mathrm{Eu}^{2+}=0.6 / 1.1 / 1.0$ for pc-LED sample 3.

Then a phosphor paste was made by dispersion of each of the above phosphor mixtures in translucent silicone resin at a concentration of $0.2-1.3 \mathrm{vol} \%$. Finally, a pc-LED was fabricated by encapsulation of an LED chip on a white substrate with the phosphor paste, which was allowed to harden at $150^{\circ} \mathrm{C}$ for $90 \mathrm{~min}$.

Determination of crystal structures. SR-XRD measurements were performed at the Japan Synchrotron Radiation Research Institute, SPring-8, with synchrotron orbit radiation on the BL02B1 and BL02B2 beamlines at a wavelength of ca. $0.35 \AA$ for the single crystals and a wavelength of ca. $0.80 \AA$ for the $\mathrm{Eu}^{2+}$-doped powder samples. The initial atomic positions of $\mathrm{Cl} \_\mathrm{MS}$ were determined directly from the data for the undoped single crystal (see Supplementary Methods).

Optical measurements. The luminescence spectra under continuous excitation and the excitation spectra of the phosphor samples were measured at room temperature with a multichannel optical analyser (PMA C5966-31, Hamamatsu Photonics). Time-resolved luminescence spectra were measured with a pulsed 266-nm laser (CFR 400, Quantel), with an excitation duration of $10 \mathrm{~ns}$ and a repetition rate of $10 \mathrm{~Hz}$. IQEs and absorptions were obtained from luminescence spectra with 407-nm laser-diode excitation, using a phosphor sample mounted in an integrated sphere. The detector employed a spectrometer (CAS 140B-152, Instrument Systems). This measurement system had two key features. First, the energy density of the excitation beam with which the sample was irradiated was high. The energy density was as high as actually mounted on an LED chip. Second, the measurement time was short, because a charge-coupled device (CCD) image sensor array was used as a detector. Consequently, we could measure the IQEs without the influence of thermal quenching by the Stokes shift. The integrating sphere had a diameter of $150 \mathrm{~mm}$ and three ports with diameters of $25 \mathrm{~mm}$. The sample was placed in the integrating sphere through the bottom port, and excitation light was directed onto the sample from the upper port. Emitted light was directed to the spectrometer through an optical receiver installed in the middle port. We obtained data by gathering the reflections generated by irradiation of the sample with a laser-diode beam (power, $10.2 \mathrm{~mW}$ at $407 \mathrm{~nm}$; irradiation time, $10 \mathrm{~ms}$; irradiated area, $1 \mathrm{~mm}$ diameter). The detector of the CAS 140B-152 spectrometer was the CCD array, which has 1,024 CCD elements. The wavelength band that could be measured was between $375-785 \mathrm{~nm}$. The reflection spectrum of the $\mathrm{BaSO}_{4}$ powder without absorption was used as a reference for calculation of the IQE and the absorption.

The CAS 140B-152 spectrometer was also used to obtain luminous flux, colour coordinates and colour temperature from a pc-LED operated in an integrated sphere.

Table 1 | EFG tensor elements $\left(V_{\mathrm{xx}}, \boldsymbol{V}_{\mathrm{yy}}, \boldsymbol{V}_{\mathrm{zz}}\right)$ and asymmetric parameter $\boldsymbol{\eta}$ from DFT calculations.

\begin{tabular}{|c|c|c|c|c|}
\hline & \multicolumn{2}{|c|}{ EFG tensor elements $\left(10^{21} \mathrm{~V} \mathrm{~m}^{2}\right)$} & \multicolumn{2}{|c|}{ Asymmetric parameter } \\
\hline & $v_{\mathbf{x x}}$ & $V_{\text {yy }}$ & $v_{z z}$ & $\eta$ \\
\hline Sr1 (M(2) site) & 9.13 & -6.10 & -3.04 & 0.34 \\
\hline Sr3 (M(3) site) & 1.74 & 7.20 & -8.94 & 0.61 \\
\hline
\end{tabular}




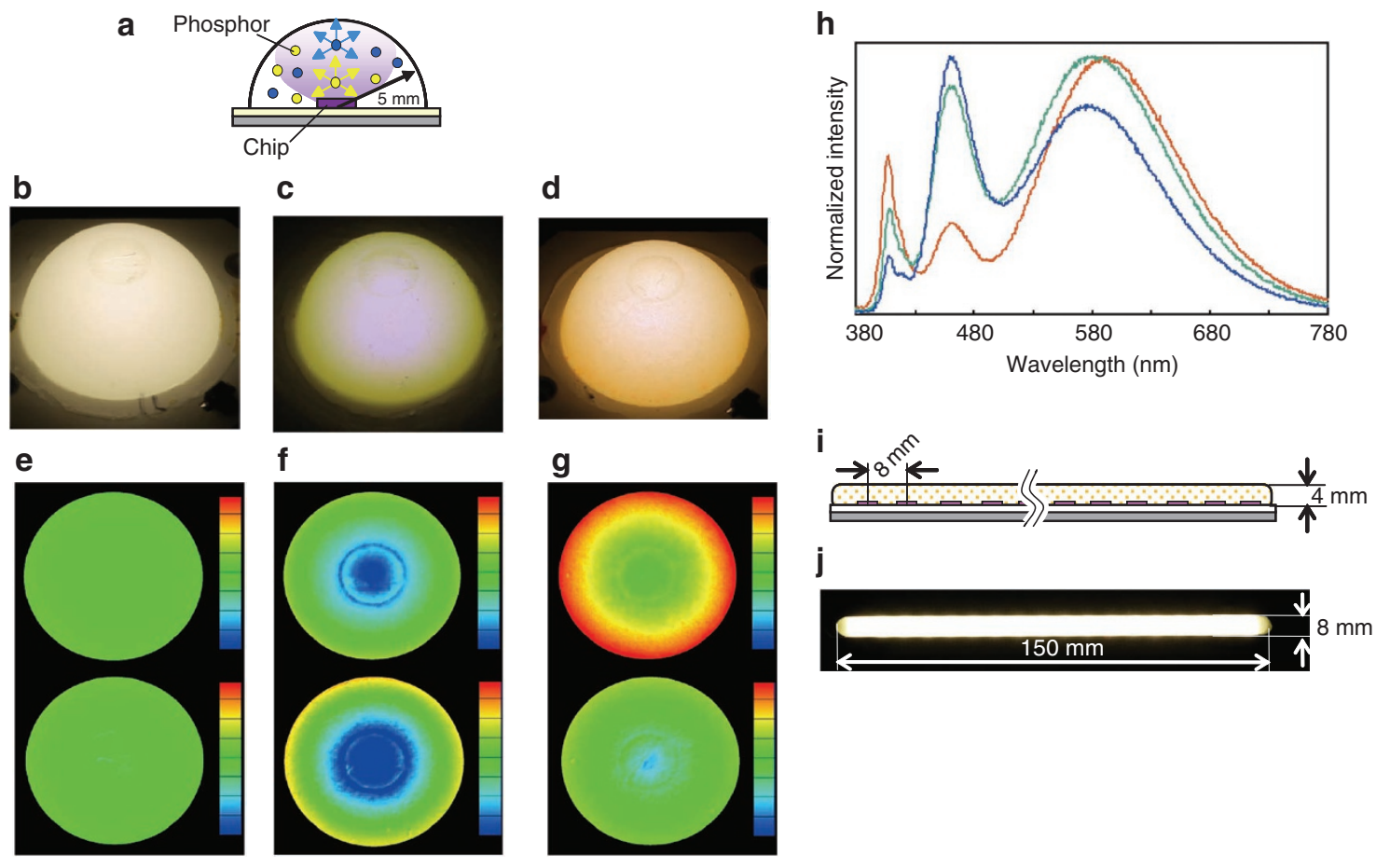

Figure 5 | Structure and performance of fabricated pc-LEDs. (a) Structure of fabricated pc-LEDs. (b-d) Images of emitting fabricated pc-LEDs with different phosphors and chips. Sample 1 consists of $\left(\mathrm{Ca}_{0.37} \mathrm{Sr}_{0.53} \mathrm{Eu}_{0.10}\right)_{7}\left(\mathrm{SiO}_{3}\right)_{6} \mathrm{Cl}_{2}$ and APT:Eu ${ }^{2+}$ coupled with a violet chip $\left(\lambda_{\mathrm{p}}=405 \mathrm{~nm}\right)$ in $\mathbf{b}$; sample 2 consists of YAG:Ce ${ }^{3+}$ coupled with a blue chip $\left(\lambda_{p}=460 \mathrm{~nm}\right)$ in $\mathbf{c}$; and sample 3 consists of a mixture of APT:Eu ${ }^{2+}, \beta-S i A I O N: E u^{2+}$ and S-CASN:Eu ${ }^{2+}$ coupled with a violet chip $\left(\lambda_{p}=405 \mathrm{~nm}\right)$ in $\mathbf{d}$. (e-g) Emission colour distribution in emission areas of sample 1 in e, sample 2 in $\mathbf{f}$ and sample 3 in $\mathbf{g}$. The upper pictures show the distribution of $C_{x}$ values used for Commission Internationale de l'Eclairage chromaticity, and the lower pictures are the $C_{y}$ values. The scale bars for $C_{x}$ represent the same chromaticity range in all the panels. The minimum (blue) value is 0.277 , and the maximum (red) value is 0.443 . The scale bars for $C_{y}$ represent the same chromaticity range in all the panels. The minimum (blue) value is 0.294 , and the maximum (red) value is 0.460 . The operation current was $350 \mathrm{~mA}$. (h) Luminescence spectra of Cl_MS:Eu ${ }^{2+}$ pc-LEDs with different colour temperatures, operated at $350 \mathrm{~mA}$. The orange curve indicates 3,010 K, the green curve 4,100 K and the blue curve 5,070 K. (i) Schematic illustration of a line-shaped module. (j) Image of the emitting line-shaped module.

A two-dimensional colour analyser (CM-1500M, Minolta) was used to obtain the luminance and emission colour distribution on the pc-LEDs.

\section{References}

1. Pimputkar, S., Speck, J. S., DenBaars, S. P. \& Nakamura, S. Prospects for LED lighting. Nat. Photon 3, 180-182 (2009).

2. Taguchi, T. Present status of energy saving technologies and future prospect in white LED lighting. IEEJ Trans. 3, 21-26 (2008).

3. Bando, K., Sakano, K., Noguchi, Y. \& Shimizu, Y. Development of high-bright and pure-white LED lamps. J. Light Vis. Env. 22, 2-5 (1998).

4. Narukawa, Y. et al. Ultra-high efficiency white light emitting diodes. Jpn J. Appl. Phys. 45, L1084-L1086 (2006).

5. Narukawa, Y. et al. Successful fabrication of white light emitting diodes by using extremely high external quantum efficiency blue chips. Phys. Stat. Sol. A 205, 1081-1085 (2008).

6. Kasahara, T. et al. Discomfort glare caused by white LED light sources. J. Light Vis. Env. 30, 95-103 (2006)

7. Taguchi, T. Present status of white LED lighting technologies in Japan. J. Light Vis. Env. 27, 131-139 (2003).

8. Hao, Z. et al. White light emitting diode by using $\alpha-\mathrm{Ca}_{2} \mathrm{P}_{2} \mathrm{O}_{7}: \mathrm{Eu}^{2+}{ }^{2+} \mathrm{Mn}^{2+}$ phosphor. Appl. Phys. Lett. 90, 261113 (2007).

9. Nishida, T., Ban, T. \& Kobayashi, N. High-color-rendering light sources consisting of a 350-nm ultraviolet light-emitting diode and three-basal-color phosphors. Appl. Phys. Lett. 82, 3817-3819 (2003).

10. Uchida, Y. \& Taguchi, T. Lighting theory and luminous characteristics of white light-emitting diodes. Opt. Eng. 44, 124003 (2005).

11. Takahashi, K. et al. Luminescence properties of blue $\mathrm{La}_{1-\mathrm{x}} \mathrm{Ce}_{\mathrm{x}} \mathrm{Al}\left(\mathrm{Si}_{6-\mathrm{z}} \mathrm{Al}_{\mathrm{z}}\right)$ $\left(\mathrm{N}_{10-\mathrm{z}} \mathrm{O}_{\mathrm{z}}\right)(\mathrm{z} \sim 1)$ oxynitride phosphors and their application in white lightemitting diode. Appl. Phys. Lett. 91, 091923 (2007).

12. Xie, R. J. et al. Strong green emission from $\alpha$-SiAlON activated by divalent Ytterbium under blue light irradiation. J. Phys. Chem. B 109, 9490-9494 (2005).
13. Hirosaki, $\mathrm{N}$ et al. Characterization and properties of green-emitting $\beta$-SiAlON: $\mathrm{Eu}^{2+}$ powder phosphors for white light-emitting diodes. Appl. Phys. Lett. 86, 211905 (2005).

14. Fukuda, Y., Ishida, K., Mitsuishi, I. \& Nunoue, S. Luminescence properties of $\mathrm{Eu}^{2+}$-doped green-emitting Sr-sialon phosphor and its application to white light-emitting diodes. Appl. Phys. Exp. 2, 012401 (2009).

15. Park, J. K. et al. Application of $\mathrm{Ba}^{2+} \cdot \mathrm{Mg}^{2+}$ co-doped $\mathrm{Sr}_{2} \mathrm{SiO}_{4}:$ Eu yellow phosphor for white-light-emitting diodes. J. Electrochem. Soc. 152, H121-H123 (2005)

16. Zeuner, M., Hintz, F. \& Schnick, W. Low temperature precursor route for highly efficient spherically shaped LED-phosphors $\mathrm{M}_{2} \mathrm{Si}_{5} \mathrm{~N}_{8}: \mathrm{Eu}^{2+}(\mathrm{M}=\mathrm{Eu}, \mathrm{Sr}, \mathrm{Ba})$. Chem. Mater. 21, 336-342 (2009).

17. Krams, R. M. et al. Status and future of high-power light-emitting diodes for solid-state lighting. J. Disp. Tech. 3, 160-175 (2007).

18. Taguchi, T. Developing white LED lighting systems and its technological roadmap in Japan. J. Light Vis. Env. 30, 177-182 (2006).

19. Craford, M. G. High power LEDs for solid state lighting: status, trends and challenges. J. Light Vis. Env. 32, 58-62 (2008).

20. Fukui, T. et al. Superior illuminant characteristics of color rendering and luminous efficacy in multilayered phosphor conversion white light sources excited by near-ultraviolet light-emitting diodes. Jpn J. Appl. Phys. 48, 11210 (2009).

21. Notzold, D., Wulff, H. \& Herzog, G. Structural and optical properties of the system $(\mathrm{Ca}, \mathrm{Sr}, \mathrm{Eu})_{5}(\mathrm{PO} 4)_{3}$ Cl. Phys. Stat. Sol. B 191, 21-30 (1995).

22. Akella, A. \& Keszler, D. A Structure and $\mathrm{Eu}^{2+}$ luminescence of dibarium magnesium orthoborate. Mater. Res. Bull. 30, 105-111 (1995).

23. Blasse, G., Wanmarker, W. L., Vrugt, J. W. \& Bril, A. Fluorescence of $\mathrm{Eu}^{2+}$ activated silicates. Philips Res. Rep. 23, 189-200 (1968).

24. Kodama, N., Sasaki, N., Yamaga, M. \& Masui, Y. Long-lasting phosphorescence of $\mathrm{Eu}^{2+}$ in melilite. J. Lumin. 94-95, 19-22 (2001).

25. Burrus, H. L., Nicholson, K. P. \& Rooksby, H. P. Fluorescence of Eu ${ }^{2+}$-activated alkaline earth halosilicates. J. Lumin. 3, 467 (1971). 
26. Lin, H., Liu, X. R. \& Pun, E. Y. B. Sensitized luminescence and energy transfer in $\mathrm{Ce}^{3+}$ and $\mathrm{Eu}^{2+}$ codoped calcium magnesium chlorosilicate. Opt. Mater. 18, 397-401 (2002).

27. Zhang, X. \& Liu, X. Luminescence properties and energy transfer of $\mathrm{Eu}^{2+}$ doped $\mathrm{Ca}_{8} \mathrm{Mg}\left(\mathrm{SiO}_{4}\right)_{4} \mathrm{Cl}_{2}$ phosphors. J. Electrochem. Soc. 139, 622 (1992).

28. Barkley, M. C., Downs, R. T. \& Yang, H. Structure of walstromite, $\mathrm{BaCa}_{2} \mathrm{Si}_{3} \mathrm{O}_{9}$, and its relationship to $\mathrm{CaSiO}_{3}$-walstromite and wollastonite-II. Am. Mineral. 96, 797-801 (2011).

29. Park, J. K. et al. White light-emitting diodes of $\mathrm{GaN}$-based $\mathrm{Sr}_{2} \mathrm{SiO}_{4}$ : Eu and the luminescent properties. Appl. Phys. Lett. 82, 683-685 (2003).

30. Uheda, K. et al. Luminescence properties of a red phosphor, $\mathrm{CaAlSiN}_{3}: \mathrm{Eu}^{2+}$, for white light-emitting diodes. Electrochem. Solid State Lett. 9, H22-H25 (2006).

31. Kijima, N. et al. New green and red phosphors for white LEDs. J. Light Vis. Env. 32, 202-207 (2008).

32. Kresse, G. \& Furthmüller, J. Efficient iterative schemes for ab initio total-energy calculations using a plane-wave basis set. Phys. Rev. B 54, 11169-11186 (1996).

33. Blöchl, P. E. Projector augmented-wave method. Phys. Rev. B 50, 17953-17979 (1994)

34. Blaha, P., Schwarz, K., Madsen, G. K. H., Kvasnicka, D. \& Luitz, J. WIEN2k, An Augmented Plane WaVe Plus Local Orbitals Program for Calculating Crystal Properties (Vienna University of Technology, 2001).

35. H.Madsen, G. K., Blaha, P., Schwarz, K., Sjöstedt, E. \& Nordström, L. Efficient linearization of the augmented plane-wave method. Phys. Rev. B 64, 195134 (2001).

36. Perdew, J. P., Burke, K. \& Ernzerhof, M. Generalized gradient approximation made simple. Phys. Rev. Lett. 77, 3865-3868 (1996).

37. Yen, M. W., Shionoya, S. \& Yamamoto, H. Phosphor Handbook 2nd edn, Ch. 2, 35-47 (CRC press, 2006).

38. Yoshida, H. et al. Experimental and theoretical investigations for excitation properties of $\mathrm{Ba}_{1-\mathrm{x}} \mathrm{Eu}_{\mathrm{x}} \mathrm{MgAl}_{10} \mathrm{O}_{17}$. J. Electrochem. Soc. 154, J196-J200 (2007).

39. Yamamoto, H., Seki, S. \& Ishiba, T. The Eu site symmetry in $\mathrm{AEu}\left(\mathrm{MoO}_{4}\right)_{2}$ $(\mathrm{A}=\mathrm{Cs}$, or $\mathrm{Rb})$ generating saturated red luminescence. J. Solid State Chem. 94, 396-403 (1991).
40. Hwang, K., Hwangbo, S. \& Kim, J. Sol-gel synthesis of red-emitting $\mathrm{LiEuW}_{2} \mathrm{O}_{8}$ powder as a near-ultraviolet convertible phosphor. Ceram. Int. 35, 2517-2519 (2009).

41. CIE. Discomfort glare in interior lighting. CIE Tech. Rep. 117-1995 (CIE Publications, (1995)).

\section{Acknowledgements}

This research was partially supported by the Ministry of Education, Culture, Sports, Science and Technology Grant-in-Aid for Scientific Research (A), no. 23244074, and the Scientific Research on Priority Areas, no. 20110003. The synchrotron radiation experiments performed at SPring- 8 were supported by the Japan Synchrotron Radiation Research Institute. H.H. acknowledges funding from the JSPS FIRST project and MEXT Element Strategy project.

\section{Author contributions}

Y. Shinomiya, T.I., K.E., Y. Sasaki, Y.M. and H.D. synthesized materials and fabricated pc-LEDs. S.A., E.N., M.S. and H.S. carried out SR-XRD measurements and structural analysis. Y. Shinomiya, T.I., K.E., Y. Sasaki, Y.M. and S.M. carried out PL measurements. S.M. and H.H. calculated EFG by DFT. H.D., Y. Shinomiya, S.A., S.M., H.S. and H.H. participated in discussions of the data and the writing of the paper.

\section{Additional information}

Supplementary Information accompanies this paper at http://www.nature.com/ naturecommunications

Competing financial interests: The authors declare no competing financial interests.

Reprints and permission information is available online at http://npg.nature.com/ reprintsandpermissions/

How to cite this article: Daicho, H. et al. A novel phosphor for glareless white lightemitting diodes. Nat. Commun. 3:1132 doi: 10.1038/ncomms2138 (2012). 